\title{
Perigraft seroma mimicking cardiac tamponade after implantation of Berlin Heart EXCOR device in an infant
}

\author{
Fabrizio Gandolfo, MD, Gianluca Brancaccio, MD, PhD, Aurelio Secinaro, MD, and \\ Antonio Amodeo, MD, Rome, Italy
}

Development of serous fluid leakage around polytetrafluoroethylene (PTFE) grafts used to perform systemicto-pulmonary arterial shunt (modified Blalock-Taussig shunt) has been reported previously. ${ }^{1}$ Occasionally, an excessive amount of serous fluid will persistently drain through the interstices of the fabric.

We report an unusual case of pericardial seroma in an infant developing around the outflow cannula of a new model of Berlin Heart left ventricular assistance device (EXCOR; Berlin Heart GmbH, Berlin, Germany), which includes a PTFE component. This seroma led to a initial diagnosis of cardiac tamponade and necessitated surgical revision.

\section{CLINICAL SUMMARY}

In a 4-month-old female infant, weighing $5.5 \mathrm{~kg}$ and affected by a severe form of dilative cardiomyopathy, a 10-mL EXCOR left ventricular assistance device was implanted. The inflow cannula was positioned in the apex of the left ventricle, and a newly designed outflow cannula was placed in the ascending aorta. The aortic cannula features a terminal part (near the aortic anastomosis) made of PTFE graft, which is intended to allow a more physiologic curvature around the heart and to prevent traction on the aortic suture.

Cardiopulmonary bypass with induced ventricular fibrillation at $30^{\circ} \mathrm{C}$ lasted 131 minutes. The procedure was performed uneventfully.

The child was admitted to the intensive care unit on a regimen of epinephrine $(0.05 \mu \mathrm{g} /[\mathrm{kg} \cdot \mathrm{min}])$, milrinone $(1 \mu \mathrm{g} /[\mathrm{kg} \cdot \mathrm{min}])$, and dopamine $(3 \mu \mathrm{g} /[\mathrm{kg}$. min]). No excessive bleeding was noted in the following days.

\footnotetext{
From the Department of Cardiac Surgery, Ospedale Pediatrico Bambino Gesù, Rome, Italy.

Disclosures: Authors have nothing to disclose with regard to commercial support.

EXCOR is a registered trademark of Berlin Heart GmbH, Berlin, Germany.

Received for publication July 2, 2012; revisions received Aug 15, 2012; accepted for publication Sept 12, 2012; available ahead of print Oct 10, 2012.

Address for reprints: Antonio Amodeo, MD, Department of Cardiac Surgery, Ospedale Pediatrico Bambino Gesù, Piazza S.Onofrio, 5- 00165 Rome, Italy

(E-mail: antonioamodeo@yahoo.it).

J Thorac Cardiovasc Surg 2013;145:e3-4

$0022-5223 / \$ 36.00$

Copyright (c) 2013 by The American Association for Thoracic Surgery

http://dx.doi.org/10.1016/j.jtcvs.2012.09.016
}

Echocardiography performed during first days after EXCOR implantation showed a normal right ventricular function. In the ensuing postoperative course, however, progressive right ventricular dysfunction was demonstrated by ultrasonographic studies. Central venous pressure rose to $15-20 \mathrm{~mm} \mathrm{Hg}$, with clinical evidence of superior vena cava syndrome. Medical treatment for right ventricular failure was started, without clinical improvement.

Cardiac catheterization was then performed. A mean gradient between the superior vena cava and the right atrium of $16 \mathrm{~mm} \mathrm{Hg}$ was measured; however, neither echocardiography nor angiography was able to reveal the etiology of the superior vena cava obstruction.

Two weeks from implantation, a contrast computed tomographic scan with 3-dimensional imaging was performed. The 3-dimensional volumetric acquisition was instrumental in detailing extrinsic compression of the superior vena cava by a high-density fluid collection around the inflow cannula, contouring the right ventricle as well (Figure 1). Direct compression from the arterial cannula itself was ruled out (Figure 2).

Surgical reexploration was conducted the next day, and a huge quantity of semisolid, yellowish material was found around the cannula and the right ventricle. After mass removal, central venous pressure immediately fell to 7-8 mm Hg. No signs of infection were observed.

Histologic examination confirmed the suspicion of serumlike material, leading to the eventual diagnosis of seroma, probably generated by the PTFE graft. Pericardial effusion has not recurred on ultrasonographic follow-up. Drainage production was almost $20 \mathrm{~mL}$ serum in the first 4 days, and removal was performed after 1 week.

The patient underwent transplant 1 month later. After an uncomplicated hospital course, she was discharged in good clinical status.

\section{DISCUSSION}

Because of the lack of heart donors in the infant population, ventricular assist devices may remain in place for several months, leading to multiple acute and chronic complications. Serum leakage in the pediatric population treated with a PTFE graft has been described previously, particularly after systemic-to-pulmonary 


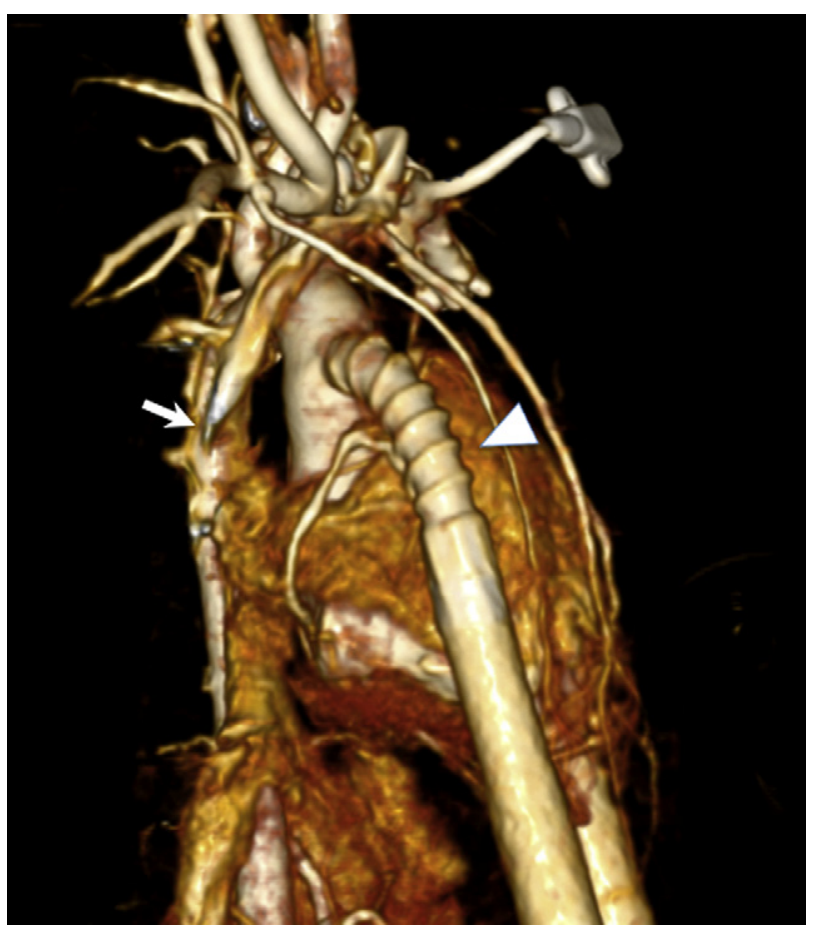

FIGURE 1. The 3-dimensional volume rendering shows superior vena cava obstruction (arrow) at the junction with the right atrium. Between the superior vena cava and the inflow cannula (arrowhead), there is a nonenhancing gap.

arterial shunts. $^{2}$ A perigraft seroma is defined as a sterile collection of fluid from a nonsecretory wall surrounding a shunt. One of the most widely accepted theories is that handling of the PTFE graft causes leakage because of a change from a hydrophobic state to a hydrophilic one.

In our case, progressive elevation of central venous pressure and mild to moderate right ventricular dysfunction, together with unclear information from echocardiography, prompted us to treat the patient for right ventricular dysfunction. Only computed tomographic scan techniques revealed the extrinsic compression of the superior vena cava and possibly the right ventricle. Surgical revision became then the obvious option, ${ }^{3}$ with immediate relief of symptoms. The roles of computed tomography and magnetic resonance imaging in detecting this complication after the Blalock-Taussig shunt have been described as well. ${ }^{4,5}$ We postulate that

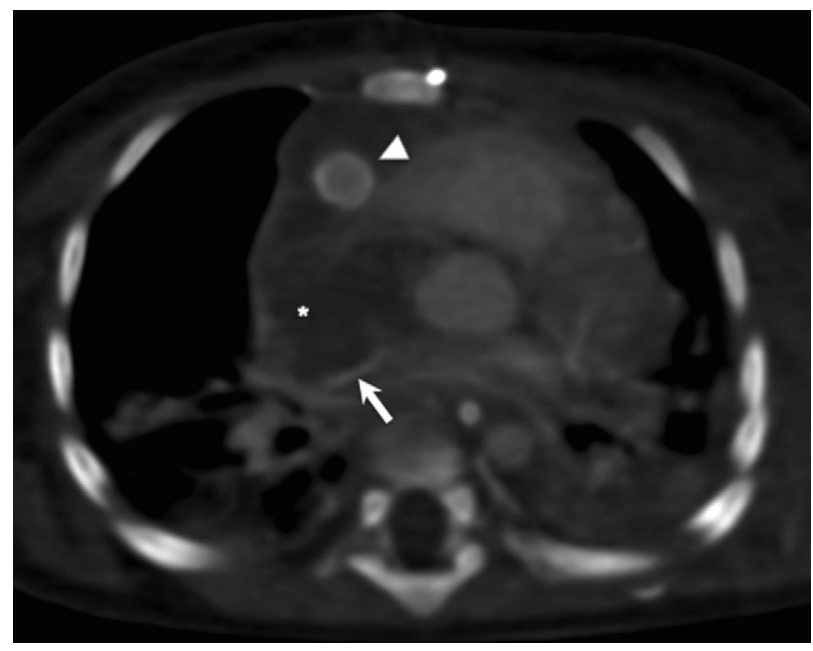

FIGURE 2. The 2-dimensional axial view reveals the presence of a fluid collection (star) surrounding the polytetrafluoroethylene aortic cannula (arrowhead) and right-sided chambers causing an extrinsic compression of the superior vena cava (arrow).

serum leakage stops for a protective inner panel formation inside the cannula a few weeks after implantation.

In this early experience with the EXCOR device with an aortic cannula of new design, a major complication occurred. Its possibility must therefore be kept in mind. We wish to stress the important role of the computed tomographic scan study in reaching the correct diagnosis. We also suggest leaving chest drains in place for at least 2 days after none or minimal fluid production to prevent seroma formation, which is our policy in Blalock-Taussig shunts.

\section{References}

1. van Rijn RR, Berger RM, Lequin MH, Robben SG. Development of a perigraft seroma around modified Blalock-Taussig shunts: imaging evaluation. AJR Am J Roentgenol. 2002;178:629-33.

2. Sahoo M, Sahu M, Kale S, Saxena N. Serous fluid leakage following modified Blalock-Taussig operation using PTFE grafts. Indian Heart J. 2001;53: 328-31.

3. Demircin M, Doğan R, Ozkan M, Ozsoy F, Kuzgun E, Güvener M. Perigraft seroma complicating the modified Blalock-Taussig shunts: two consecutive instances with review of literature. Turk J Pediatr. 2004;46:275-8.

4. Duerinckx A, Atkinson D, Klitzner TS, Perloff J, Drinkwater D, Laks H. MR imaging of surgical complications of systemic-to-pulmonary artery shunts. Magn Reson Imaging. 1996;14:1099-105.

5. Rudd SA, McAdams HP, Cohen AJ. Mediastinal perigraft seroma: CT and MR imaging. J Thorac Imaging. 1994;9:120-2. 